\title{
Applying IDC theory to education in the Alps region: a response to Chan et al.'s contribution
}

\author{
Pierre Dillenbourg ${ }^{*}$, Kevin Gonyop Kim, Jauwairia Nasir, Shuqing Teresa Yeo, and Jennifer K. Olsen
}

${ }^{*}$ Correspondence: pierre.dillenbourg@epfl.ch Computer-Human Interaction for Learning and Instruction (CHILI) laboratory, École Polytechnique Fédérale de Lausanne (EPFL), Route Cantonale, 1015 Lausanne,

Switzerland

\begin{abstract}
In this paper, we present a response to the Interest-Driven Creator (IDC) theory from a European perspective. Specifically, we raise six questions intended to start a dialog with respect to IDC theory's placement in existing learning theories, its adoption in educational systems, and how it can be influenced by emerging learning technologies and digitalization, which is currently a driving force in the Alps region. By referring to our own work in vocational education and classroom orchestration, we demonstrate how IDC can begin to play a part in guiding innovations and its potential impact on education both in and outside of Asia. With respect to digitalization, rather than allowing technological innovations to fully guide educational decisions, we call for IDC theory to be part of the conversation to help guide future educational designs.
\end{abstract}

Keywords: Interest-Driven Creator (IDC) theory, Learning theory, Vocational education, Learning analytics, Classroom orchestration

\section{Introduction}

In Chan et al.'s seminal contribution, they present Interest-Driven Creator (IDC) theory as a theory of learning design that, if adopted, will develop students' twenty-first century competencies and bring a focus to the learning process rather than only focusing on the learning outcomes. IDC theory is grounded in a need to reshape educational systems in Asia, and in our response, we reflect on IDC from an European perspective, even if three of the authors come from Asia. Specifically, we examine the theory from the point of view of how it fits into the broader spectrum of learning theories, how technology and education may foster its adoption, and the broader impact of the theory. We structure our response into six questions and highlight the relevance to the peculiarities of the vocational education and training (VET) systems that exists in a few countries surrounding the Alps. As the VET systems are wellestablished foundations of the educational systems in this area, this perspective allows us to contemplate the IDC theory from social and cultural perspectives that influenced its creation. Particularly, our answers articulate the IDC theory with our own conceptual contribution such as the "Erfarraum" model and the classroom orchestration framework.

(c) The Author(s) 2019 Open Access This article is distributed under the terms of the Creative Commons Attribution 4.0 International License (http://creativecommons.org/licenses/by/4.0/), which permits unrestricted use, distribution, and reproduction in any medium, provided you give appropriate credit to the original author(s) and the source, provide a link to the Creative Commons license, and indicate if changes were made. 


\section{Does IDC constitute a learning theory?}

The answer to this question depends upon our personal understanding of what a learning theory should be. We see at least three levels of understanding of this term: as an explainable prediction, as a conceptual framework, and as a political message.

Let us start with the third understanding. IDC theory clearly conveys a political message to policy makers in Asian educational systems. It is a plea for a more equilibrated development of children by fostering a broader range of skills than those measured through large scale tests. This plea is especially relevant in Asian countries where the culture of tests is extremely salient (Ho 2009), even if Singapore is already moving away from this culture (Koh et al. 2012; Lee et al. 2016). Actually, it also applies to many Western countries where the commendable quest for effectiveness in education is often perverted into more testcentric education. A common terminological fallacy pushes educational policies to slowly slide from evidence-based to test-based approaches. But can a learning theory have such a cultural and political impact, namely change educational practices that have become so deeply entangled in the national culture? In several education systems, previous attempts of suppressing grades have raised instant opposition from parents because a qualitativeonly assessment of their children's skills blurred their understanding of their children's school performance. However, the acceptability of the IDC theory proposition is much higher than any radical attempt to change school practices through suggesting the cohabitation of a culture of school examination with a culture of personal development around twenty-first century skills. Will this diplomatic strategy create the momentum necessary for bending the Asian education culture? The future will tell.

We will now consider the first understanding of what a learning theory is: an actionable prediction of how learning occurs. In applied research, these theories can be phrased as the prediction of how some learning activities produce specific learning processes or outcomes where the outcome can be as simple as the fact that learning has occurred. For instance, the theory of "advanced organizers" predicts that pre-activating relevant mental schemata enhances the acquisition of new knowledge (Ausubel 1960). The "contrasting cases" theory predicts that telling the concepts to students before asking them to solve problems leads to lower transfer (Schwartz et al. 2011). These predictions are only actionable if they suggest concrete instructional design choices and provide an explanation of the causal mechanisms behind the effects. Understanding the process is a necessary condition to be able to instantiate the general principles within the specific domain to be learned (e.g., physics versus grammar). Although IDC theory aims to have predictive power and to be actionable, further developments are required to reach the level of operationalization described above: "an immediate challenge for IDC theory is for it to evolve as a design theory," as recognized by the authors (Chan et al. 2018, p.453).

Finally, for our second understanding of what a learning theory entails, we could question whether advanced organizers or contrasting cases are learning theories or, more modestly, instructional design principles. We may expect a theory to be a conceptual framework and include multiple design principles and concepts. For example, we do not qualify the process of reinforcement as a theory but as one concept within the behaviorist theory. The same distinction can be made between accommodation and constructivism or between internalization and socio-cultural theories. Clearly, the authors place IDC theory at this level by calling it a "macro-level" theory, and some flavors of developmental 
stages of Piaget can be found within each of the three IDC loops. For example, IDC describes how cuing leads to routine then to harmony, a very Asian terminology for talking about education.

In summary, IDC has some of the ingredients that constitute theories, concepts, predictions and explanations, integrated into a global framework, and conveying some political message.

\section{Does education need yet another learning theory?}

A theory is a lens that brings a certain way to look at educational situations, a way to frame educational questions, or a certain perspective on data analyses. The IDC lens sheds light on concepts such as interest and creation. Within IDC theory, interest is broken into triggering, immersing, and extending, which closely aligns with the three-factor situational interest model (Linnenbrink-Garcia et al. 2010) and is composed of triggering, maintained feeling, and maintained value. Yet, interest within the IDC theory seems to go beyond just situational interest and also encompasses some aspects of individual interest (Hidi and Renninger 2006) and intrinsic motivation (Vallerand 1997) but with a different flavor. The way we read their notion of interest seems less related to the perceived usefulness of a specific skill to be acquired or content to be learned but rather as some kind of self-directed drive for learning, some curiosity to discover new things. This flavor is salient in the IDC concept of "lifelong interest-driven creators." For creation, the notion is not very present in the learning theories we previously mentioned, which is a pity. It is, however, present in a theory such as "productive failure" which stresses the fact that producing more than a single solution during the unproductive problem-solving phases is a condition for success and is a habit that must be practiced (Kapur 2008). IDC theory also stresses this need for students to generate multiple creations to achieve creativity. Interestingly, when regarded as a whole, IDC theory articulates concepts that could be viewed as an antinomy, such as interest/creation/passion on the one hand but routines/habits on the other although the theory seems to emphasize that the interest/creation/passion should become the routines/habits. We expect the tension between these two poles to become a strength of the IDC theory, an opportunity to create new designs. So there is some novelty within this theory, which justifies the usefulness of a new theory.

However, there is a drawback in producing new theories: educators seem to consider theories as mutually exclusive and, therefore, subscribe to one and reject others in the same way they subscribe to a single political party or a single religious community. Instead, our research on classroom orchestration is trying to promote the integration of activities inspired by different theories in one pedagogical scenario. In this ecumenical vision, a designer or teacher could start a lesson by a constructivist activity, followed by a collaborative activity, lecture, and ending with a mastery learning activity to best align with the pedagogical goals of each phase. Orchestration graphs (Dillenbourg 2015) operationalize the integration of heterogeneous activities into a consistent pedagogical workflow. We follow Wenger's approach of theories as "plug \& play" modules: "Social theories do not compete in terms of being true or false; they compete in terms of the usefulness of their perspective in enabling certain types of accounts about the human world" (Wenger-Trayner 2013, p.3). Along this line of thinking, we would ask where the IDC theory could be plugged into and what it would bring. A natural fit seems to exist 
with inquiry-based learning or so-called "constructionists" activities, which the IDC theory pragmatically enriches with steps such as "imitating" or "routine" that may not have received enough attention in socio-constructivism. However, the IDC theory does not just provide guidance on how to design individual activities. It also proposes an overarching way of designing an entire lesson or, on a larger scale, an entire curriculum. Through this lens, the IDC theory may enrich the connections made between different activities by grounding the pedagogical purpose of them within the IDC loops.

\section{Will the digital revolution promote the adoption of IDC?}

The last few decades revealed the co-evolution of learning theories and information technologies. For instance, we have seen a renaissance of social theories of learning in the eighties in parallel to the sudden spread of telematics, first local networks then the Internet. Neither of these trends was the cause of the other, but their coincidence produced an exponential growth of computer-supported collaborative learning and, later on, learning communities. Before that, individual tasks were the cornerstone of both computers, then called "personal computers," and learning activities, such as mastery learning and constructivism. It should be noted that neither technologies nor learning theories have disappeared when a new one emerged, they juxtapose to teach each other, first in a competitive mode and then, as maturity develops, as complementary theories with their sphere of relevance or as mutually compatible technologies.

The relationship between the digital turn of our society and economy in the twentyfirst century and the emergence of the IDC theory is more indirect. The authors explain mostly that the digital revolution has changed the skills expected from future citizens. Memorizing a large quantity of terms or basic procedural skills becomes partly obsolete if the same performance is embedded in most digital devices. On the other hand, the skills promoted by IDC theory, especially creativity, will become central to make humans resilient to the advances of artificial intelligence. So, the digital sphere explains the need to develop new approaches to education that develop all aspects of human talent, and IDC theory belongs to this trend.

In addition to this indirect relationship between the digital turn and IDC theory, technologies are also direct enablers of IDC implementations. For example, digital environments may boost creativity by enabling learners to propose not one solution to a problem but to explore the solution space through prolific creation. In our work on training florists (Kim et al. 2019), we enable them to explore the space of possible designs of a bouquet through the use of digital variations of a real bouquet (Fig. 1). The students start from a bouquet placed in the center of the left pane. They can choose among four possible variations of the initial bouquet, namely changing the color (top left axis), the shape (top right), the spatial layout (bottom right), or the texture (bottom left) of the bouquet. The selected variation then moves to the center of the display, and four new variations are proposed again and so forth iteratively. The smaller thumbnails on the top left bar allows them to backtrack to a previous stage of the design process. On the right pane, they can explore the center bouquet in 3D and access the name of any component thanks to the BloomyPro application. Beyond the interest of letting them try multiple solutions or designs, a fundamental learning outcome is the understanding of a designing task as finding a solution in a multi-dimensional space of decisions. This may sound trivial to any scholar, especially AI researchers, but our research explores if low-achieving teenagers are 


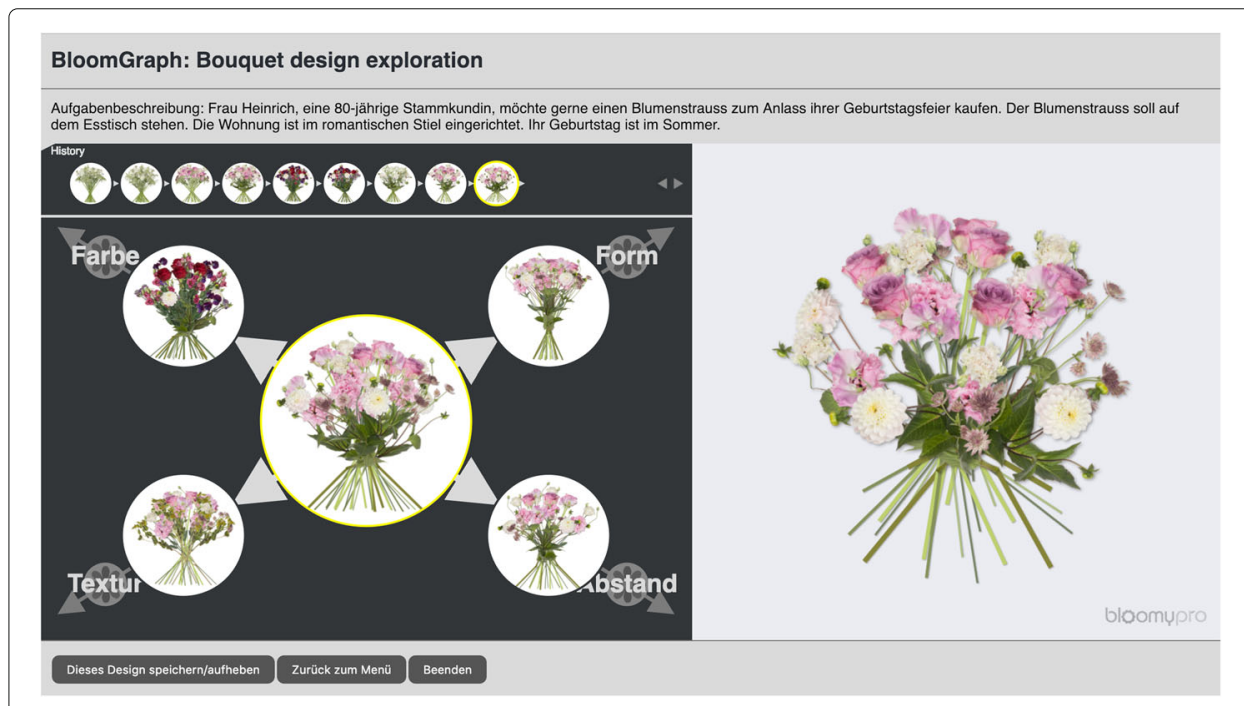

Fig. 1 A tool enabling future florists to explore the space of possible designs

also able to engage in this combinatorial process. The technology enables us to approach this combinatorial process intuitively to support learning.

Although the relationship between the digital revolution and the emergence of the IDC theory might seem indirect, they have a reciprocal relationship. The development of the skills promoted by IDC theory will be necessary in a new digital culture. In return, as shown in our example, the digital technologies can enable the implementation of the theory and eventually promote its adoption.

\section{Is IDC threatened by learning analytics?}

The question may surprise the reader, but it originates from the constraints that computational modeling imposes on learning tasks. A trend in educational research is to model computationally the learning process: applying data science methods to behavioral traces enables the adaptation of instruction, the prediction of undesirable events (drop-out, failure) and, more fundamentally, the study of learning processes (Baker and Inventado 2014). Specifically, knowledge tracing requires the matching of target skills with specific behaviors, typically correct or incorrect answers. This modeling, such as Bayesian Knowledge Tracing (Corbett and Anderson 1994), allows for knowledge assessment to occur as part of the learning process rather than through designated tests. However, the nature of skills promoted by IDC theory does not necessarily translate in terms of correct or incorrect behavior. It is not easy to capture creativity with digital sensors.

So the answer to the question in the section title is positive. Although learning analytics and IDC theory on the surface are not at odds with one another, there is the situation as described above in which a drive for more data of certain types could drive education away from the activity designs proposed in IDC preventing its adoption. However, the risk to reduce education to what can be easily quantified is minimized by recent developments in learning analytics. For instance, if one can train a machine learning model with a large set of essays classified by teachers as creative or not, then text mining methods can be used to quite accurately classify and provide student feedback (Woods et al. 2017). Moreover, new sensors and powerful computational models enable so-called multimodal 
analytics, i.e., applying data sciences methods to signals that are not captured by traditional keyboard and mouse such as gestures, gaze, body postures, and electro cardiology (Ochoa and Worsley 2016). Multimodal analytics allow for the correlation of such signals to performance, especially for skills that require a more complex measurement scale than binary. To continue the promotion of these diverse forms of learning analytics, it is important to start learning designs based in theory, IDC or any other one, and then consider how learning analytics can be used to support these activities rather than relying on the need for more data to drive the learning designs.

\section{Is IDC relevant to vocational education?}

We believe so. The previous example with florists convinces us that the IDC theory would be relevant to VET. We focus on the Swiss VET system as our experience with it has led us to observe that it has many connections with IDC theory. The Swiss VET system, similarly to the German and Austrian ones, is mostly based on a dual mode where apprentices spend 3-4 days per week in a company and 1-2 days at school with a culture focused on creation and practical skills. The jobs encompass many manual workers (e.g., carpenters, mechanics, electricians, gardeners, and bakers), salespeople, office clerks, and social/health professions. This approach concerns no less than two thirds of Swiss teenagers. Since many of these professions are endangered by the advances in robotics and artificial intelligence, the reflections that underlie the development of the IDC theory are especially relevant for VET and are at the center of multiple debates and initiatives.

The apprentice's learning path in a VET system matches several points of the three IDC loops. During their initial months at their workplace, apprentices have a low level of responsibility and they mostly practice elementary professional gestures by imitating (first step of the IDC creation loop) the examples provided by the supervisors. As they gain responsibility, their supervisors allocate them more complex tasks which require combining (second step of the creation loop) multiple basic gestures or sub-tasks. In their final year, as in the staging step (third step of the creation loop), many apprentices have to develop their masterpiece, an original demonstration of their craftsmanship and creativity, and present it to the examiners, their peers, and their community.

Another central concept that connects IDC theory and VET is the notion of meaningfulness within the extending step of the interest loop. The authors define interest as the fact that students "associate the context of their real-life situations with what they are learning" (Chan et al. 2018, p.445), which is also central to VET since skills are acquired in the context of their future use (i.e., the workplace). However, this meaningfulness is sometimes questioned in a dual system because of gaps that emerge between the skills taught at school and those practiced at the workplace. Even if the school curricula are defined by the corporate associations, these gaps exist. In terms of the nature of knowledge, more conceptual/declarative knowledge is taught at school versus more procedural/practical knowledge at the workplace. In terms of the time horizon, at the workplace, apprentices perform the tasks required by the company on a daily basis while schools have long-term ambitions for their apprentices. For example, warehouse apprentices learn how to optimize the layout of a warehouse at school, but in the workplace, this skill is practiced by their bosses and not by them. This school-workplace gap creates a breach of meaningfulness, since they are taught skills that they do not have immediate use for. To restore meaningfulness, we designed the "Erfahraum" model (translated as "experience space"), 
a pedagogical design that consists in capturing experience at the workplace in order to exploit it in the classroom. Experience has become a digital substance that is captured everyday through using the mobile phone. All pictures or videos students take at their workplace can be stored in the digital flow of their class, which acts as a social media platform where apprentices from the same class may comment and tag posts as well as annotate pictures. Teachers can exploit this experience during their lectures in order to increase meaningfulness by supporting apprentices to connect theoretical concepts with their personal experience in authentic contexts.

In our recent work on training gardeners, we provide an example of associating real-world situations and learning to create meaningfulness. For training gardeners, we developed a virtual reality application for designing and simulating garden designs where the key idea is to use a place from real-life in a digital learning activity (Fig. 2). Learners can create a 3D model of a site that they are working on by taking pictures or videos. Once a 3D model is generated, apprentices can start designing a garden in the virtual world. They can add, move, rotate, or remove plants instantly with a few clicks, walk inside the garden immersively with the virtual reality headset, and simulate seasonal change, growth of trees, and the time of the day. In this learning activity, we create meaning by using a 3D model of a real-world place for students to practice designing and try different design principles. In this way, what they learn can be better connected to their real-world practice.

The IDC theory is not just relevant to the European VET system but also relates to the situation of VET in Asia. In many Asian countries, including South Korea and Singapore, students choose or sometimes are forced to choose VET because they are not doing well in schools academically. Given the situation, it is generally the case in vocational schools that the pressure of the test-based evaluation is relatively low for students and teachers. This phenomenon is not only limited to Asia but present in many other countries in the world. As the motivation behind the IDC theory is to move away from the test-centric approach, VET can be used as an example of promising starting points and an interesting testbed based upon the connections it already has with the IDC theory. Moreover, it is also interesting to note that there is an increase in the number of students in Asia who choose VET because of their interest in the profession, not because they are failing in schools. Additionally, it is not difficult to hear about successful startups that are founded by VET graduates. We find that this shift towards the interest-driven choice of the students shares the same spirit with the authors' effort on developing IDC theory.

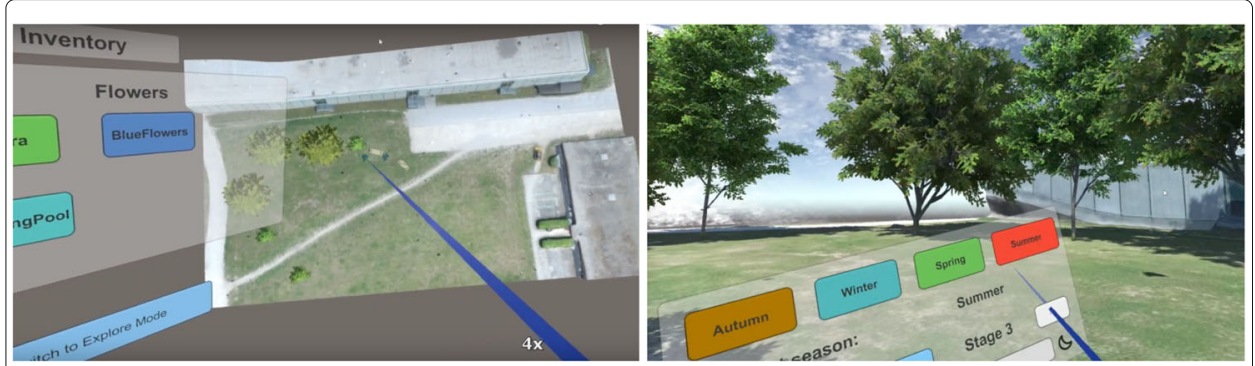

Fig. 2 A virtual reality garden designing tool for gardener apprentices. They use the 3D model of the real-world site to build a virtual garden 
In addition to the current connections between IDC theory and VET mentioned above, IDC also provides direction in regards to the future of VET. VET is facing a future of transformation due to changes in culture and society brought on by digitalization, which is impacting the skills being taught, the tools used for learning, and the educational data being collected. The core ideas presented in IDC theory can help to guide this transformation as our efforts are required to be scaled-up to impact the VET system at the cultural and societal level.

\section{Is IDC enough?}

Clearly not. IDC theory mostly informs the design of instruction, like most theories do. However, design is only one part of educational action. Design quality is a necessary condition for developing effective educational activities, but it is obviously not a sufficient condition. Any lesson, any course, any seminar requires constant adaptation from what has been planned to what actually occurs during the course of learning. Education is not an exact science. Teachers have to adapt in real time their plans to what is happening in the classroom because of behavioral, social, or cognitive factors. Technology-enhanced education has supported this adaptation to provide students with personalized instruction. Although this adaptation is primarily associated with individual learning (Aleven et al. 2016), within the computer-supported collaborative learning community there has been substantial work focusing on adaptive collaborative learning support (Magnisalis et al. 2011). It is only recently that this personalization has started to consider individual and collaborative learning together (Olsen et al. 2018). Let us stress that individual learning should be kept in balance with the need to develop social skills, which is emphasized by IDC theory and, more fundamentally, by the fact that human cognition is fundamentally social (Vygotsky 1978).

In digital systems, the decision to adapt instruction relies on the system's ability to detect that such an adaptation is needed, i.e., monitoring the learner's state. In genuine classrooms, which are very much the focus of the IDC theory, this monitoring is performed by the teacher through questioning learners and visually scanning for non-verbal cues across the classroom. Over the last few years, digital environments using teacher dashboards have provided teachers with a synthetic account of the state of their learners that they can use as additional information to decide when adaptation is needed. The data for this synthesis may be easily collected if the learners themselves use a digital learning environment in the classroom. However, these teaching prostheses will progressively extend to non-digital learning activities thanks to the advances in multimodal analytics (see the "Is IDC threatened by learning analytics?" section).

Furthermore, the deployment of educational designs encounters a diversity of events that go beyond the variations of the learners' state of knowledge or skills. In genuine classrooms, many other unexpected events may require a change of plans: two students arrive very late, the Wi-Fi network breaks down, some road work outside produces too much noise, etc. One could simply say that these situations require the teacher to redesign what has been planned. Orchestration can then be considered similar to design but in a different time frame. Several researchers have developed concepts and tools regarding classroom orchestration (VanLehn et al. 2016), i.e., the process of adapting activities "on the fly" not only on the basis of the pedagogical events but also on the basis of external constraints or events (e.g., time, noise, security). Orchestration is not a learning theory, 
it does not explain how people learn, but it emphasizes that neglecting very practical aspects of education may spoil the most perfect educational design.

We argue that it is more difficult to orchestrate a constructivist than a behaviorist sequence of activities. Similarly, we hypothesize that IDC-inspired lessons may actually be more difficult to orchestrate than traditional lectures or exercise sessions since they may include more open problem-solving activities and a richer variety of social interactions. Therefore, we recommend considering classroom orchestration within the IDC theory. Orchestration is a condition to implement IDC design principles into daily practices and a way to frame the adaptation that may be needed in real time to keep the students' practices in alignment with the interest and creation loops.

\section{Conclusions}

To conclude, we used the six questions we address in this paper to probe into the comprehensiveness of the IDC theory from a European perspective. Through these questions, we aimed to understand the lens that the IDC theory would bring to education, the relationship it has with emerging technologies, and the broader impact of the theory. As the authors point out, there are still challenges that need to be addressed within the theory. As the IDC theory currently sits, it could be understood from the perspective of three different types of learning theories. These different perspective change how IDC fits in with other theories and its influence on instructional design. To begin to put the IDC theory into practice, researchers need to be able to understand the lens that it is proposing. However, through the six questions, we can also see the potential impact that IDC theory can have on education both in and outside of Asia by shining a light on the importance of interest and creation. We use our own work in the VET system as an example of the importance of these concepts to education.

Going forward, it is important for the IDC theory to be part of the conversation to guide innovations in education. As digital education and learning analytics become the norm in education, it is important that we have our learning science theories guide their designs rather than the other way around. Without this guidance, we risk losing the balance between more complex tasks, such as collaborative learning, and more streamlined tasks, such as individual learning, as we develop for whatever is easiest and provides the most data. Additionally, it is important to learn from the cycles that we have gone through with previous learning theories. As with almost all new lesson structures, we know that it is not enough to just plan a lesson and that it will work. We need to be able to adapt to both the internal (learning) constraints and external (classroom) constraints that occur in real time through the use of classroom orchestration. By bringing IDC theory into the conversation, it can be further refined as well as help guide the discussions in a symbiotic manner.

\section{Abbreviations}

IDC: Interest-driven creator; VET: Vocational education and training

\section{Acknowledgements}

Not applicable.

\section{Authors' contributions}

PD conceived of the paper structure, formulation of the sections, and helped to draft the manuscript. KK contributed to the grounding of the paper within vocational education and cultural aspects and helped to draft the manuscript. JN and SY contributed to the cultural grounding in the paper and helped to draft the manuscript. JO helped to structure the paper, ground the paper in learning science theory, and helped to draft the manuscript. All authors read and approved the final manuscript. 


\section{Funding}

This project has received funding from the European Union's Horizon 2020 research and innovation programme under grant agreement No 765955.

Availability of data and materials

Data sharing not applicable to this article as no datasets were generated or analyzed during the current study.

Ethics approval and consent to participate

Not applicable.

\section{Consent for publication}

Not applicable.

\section{Competing interests}

The authors declare that they have no competing interests.

Received: 14 June 2019 Accepted: 25 September 2019

Published online: 11 November 2019

\section{References}

Aleven, V., McLaughlin, E.A., Glenn, R.A., Koedinger, K.R. (2016). Instruction based on adaptive learning technologies. In R.E. Mayer \& P. Alexander (Eds.), Handbook of research on learning and instruction: Routledge.

Ausubel, D.P. (1960). The use of advance organizers in the learning and retention of meaningful verbal material. Journal of educational psychology, 51(5), 267.

Baker, R.S., \& Inventado, P.S. (2014). Educational data mining and learning analytics, In Learning Analytics (pp. 61-75). New York: Springer.

Kim, K.G., Oertel, C., Dillenbourg, P. (2019). Bloomgraph: Graph-based exploration of bouquet designs for florist apprentices, In the Proceedings of the 14th European Conference on Technology Enhanced Learning (EC-TEL) (pp. 727-731). Cham: Springer.

Chan, T.-W., Looi, C.-K., Chen, W., Wong, L.-H., Chang, B., Liao, C.C., Cheng, H., Chen, Z.-H., Liu, C.-C., Kong, S.-C., et al (2018). Interest-driven creator theory: Towards a theory of learning design for asia in the twenty-first century. Journal of Computers in Education, 5(4), 435-461.

Corbett, A.T., \& Anderson, J.R. (1994). Knowledge tracing: Modeling the acquisition of procedural knowledge. User modeling and user-adapted interaction, 4(4), 253-278.

Dillenbourg, P. (2015). Orchestration Graphs. Lausanne: EPFL press.

Hidi, S., \& Renninger, K.A. (2006). The four-phase model of interest development. Educational psychologist, 41(2), $111-127$.

Ho, E.S.-c. (2009). Characteristics of east asian learners: What we learned from pisa. Educational Research Journal, $24(2), 327$.

Kapur, M. (2008). Productive failure. Cognition and instruction, 26(3), 379-424.

Koh, K.H., Tan, C., Ng, P.T. (2012). Creating thinking schools through authentic assessment: The case in singapore. Educational Assessment, Evaluation and Accountability, 24(2), 135-149.

Lee, S.-S., Hung, D., Teh, L.W. (2016). An ecological view of conceptualising change in the singapore education system. Educational Research for Policy and Practice, 15(1), 55-70.

Linnenbrink-Garcia, L., Durik, A.M., Conley, A.M., Barron, K.E., Tauer, J.M., Karabenick, S.A., Harackiewicz, J.M. (2010). Measuring situational interest in academic domains. Educational and psychological measurement, 70(4), 647-671.

Magnisalis, I., Demetriadis, S., Karakostas, A. (2011). Adaptive and intelligent systems for collaborative learning support: A review of the field. IEEE transactions on Learning Technologies, 4(1), 5-20.

Ochoa, X., \& Worsley, M. (2016). Augmenting learning analytics with multimodal sensory data. Journal of Learning Analytics, 3(2), 213-219.

Olsen, J.K., Rummel, N., Aleven, V. (2018). Co-designing orchestration support for social plane transitions with teachers: Balancing automation and teacher autonomy. In J. Kay \& R. Luckin (Eds.), Rethinking Learning in the Digital Age: Making the Learning Sciences Count, 13th International Conference of the Learning Sciences (ICLS) (pp. 1541-1542). London: International Society of the Learning Sciences.

Schwartz, D.L., Chase, C.C., Oppezzo, M.A., Chin, D.B. (2011). Practicing versus inventing with contrasting cases: The effects of telling first on learning and transfer. Journal of Educational Psychology, 103(4), 759.

Vallerand, R.J. (1997). Toward a hierarchical model of intrinsic and extrinsic motivation, In Advances in Experimental Social Psychology vol 29 (pp. 271-360). Amsterdam: Elsevier.

VanLehn, K., Cheema, S., Wetzel, J., Pead, D. (2016). Some less obvious features of classroom orchestration systems, In Educational Technologies: Challenges, Applications and Learning Outcomes (pp. 73-94). New York: Nova Science Publishers, Inc.

Vygotsky, L.S. (1978). Mind and Society: The Development of Higher Mental Processes. Cambridge: Harvard University Press.

Wenger-Trayner, E. (2013). The practice of theory: Confessions of a social learning theorist. Reframing Educational Research: Resisting the'what works' agenda. Abingdon and New York: Routledge.

Woods, B., Adamson, D., Miel, S., Mayfield, E. (2017). Formative essay feedback using predictive scoring models, In Proceedings of the 23rd ACM SIGKDD International Conference on Knowledge Discovery and Data Mining (pp. 2071-2080). Halifax: ACM.

\section{Publisher's Note}

Springer Nature remains neutral with regard to jurisdictional claims in published maps and institutional affiliations. 